\title{
Singularity Study of Sectorial Domain by Weak Formulation and Fractal Finite Element in Hamilton System
}

\author{
Kewei Ding \\ School of Civil Engineering, Anhui Jianzhu University, Hefei 230601, Anhui, P.R. China, \\ Email:dingkw@ahjzu.edu.cn
}

\begin{abstract}
The weak formulation of mixed state equations including boundary conditions are presented in polar coordinate system, mixed variational formulation is established in sectorial domain. The fractal finite element method is used to analyse the sector domain problem. The present result is exactly analogous to the Hamiltonian mechanics for a dynamic system by simulating time variable $t$ with coordinate variable $r$. The stress singularity at singular point is investigated by means of the fractal finite element method. The present study satisfies the continuity conditions of stresses and displacements at the interfaces. The principle and method suggested here have clear physical concepts. So this method would be easily popularized in dynamics analysis of elasticity.

Keywords: Hamilton system; sectorial domain; mixed equation; stress singularity; fractal finite element.
\end{abstract}

\section{Introduction}

The finite element method has experienced the development and evolution from a single mechanical variable to a number of mechanical variables. The traditional finite element method with a single displacement variable is the simplest method. Its fundamental difficulty is that it is difficult to give a smooth element function with geometric invariance and full expression, and the precision is not very high [1]. The multi-variable finite element method can not only solve the problem of first-order continuous differentiable smoothness at the junction of function curves, but also obtain the elements with good precision. However, the multi-variable finite element method is very likely to be short of rank [2]. The quasi-conforming finite element method proposed creatively by [3] does not require the stress variable to satisfy the equilibrium equation in advance, and the process of calculating the inverse matrix is omitted to obtain the higher discrete precision of the strain. The same interpolation function is selected on the element interface by the quasi-conforming finite element method and its mechanical concept is explained clearly by [4]. There is a direct relationship between the quasi - conforming element and the generalized variational principle [5] and it is proved that the quasi-conforming element is convergent and effective [5]. The advantage of the generalized conforming element method proposed by [7] is able to realize the displacement deformation compatibility between elements on the node displacement. and can ensure the convergence of the solution due to the reduction of degree of freedom.

The theory of elastic mechanics and symplectic algorithm under the Hamilton system have been unprecedentedly developed. However, there are few studies on applying the symplectic algorithm to obtain numerical solution of elastic mechanics. Considering the special properties of Hamiltonian matrix, author proposed an effective symplectic algorithm based on weak formulation of equations to solve such problems. As discussed in the previous work [8-15], weak formulation of generalized equation is the intrinsic essence of quasi-conforming finite element, and it is also the common basis of the quasi-conforming element and the Hamiltonian element. The numerical solution is the exact solution of generalized compatibility equations and satisfies the weak continuity requirement naturally.

The present paper extends such method to the singularity study of sectorial domain. Based upon the weak formulation of mixed state equations including boundary conditions, mixed variational formulation is established in sectorial domain. The stress singularity at singular point is investigated by means of the fractal finite element method.

\section{Weak Formulation}

A sectorial domain is used. Let $u$ and $w$ be the displacement in the $r$-and $\theta$-directions. Also, let $\Omega, S$ and $f_{i}$ be area, boundary line and body forces, respectively. $p_{r}$ and $p_{\theta}$ are the surface force in the radial direction and the circumferential direction, respectively. The equilibrium equation in the radial direction for $\delta \Omega$ can be written as 
$\int_{\delta S} p_{r} d s+\iint_{\delta \Omega} f_{r} d \Omega=0$

when $\bar{p}_{r}$ acting on partial boundary $S_{\sigma}$, for all area and has

$\int_{S-S_{\sigma}} p_{r} d s+\int_{S_{\sigma}} \bar{p}_{r} d s+\iint_{\Omega} f_{r} d \Omega=0$

Rewriting above equation and we have

$\int_{S} p_{r} d s+\int_{S_{\sigma}}\left(\bar{p}_{r}-p_{r}\right) d s+\iint_{\Omega} f_{r} d \Omega=0$

By means of green-formulation we can obtain

$\iint_{\Omega}\left(\frac{\partial \sigma_{r}}{\partial r}+\frac{1}{r} \frac{\partial \sigma_{r \theta}}{\partial \theta}+\frac{\sigma_{r}-\sigma_{\theta}}{r}+f_{r}\right) d \Omega+\int_{S_{\sigma}}\left(\bar{p}_{r}-p_{r}\right) d s=0$

The equilibrium equation in the tangential direction for $\delta \Omega$ can be written as

$\int_{\delta S} p_{\theta} d s+\iint_{\delta \Omega} f_{\theta} d \Omega=0$

for all area one has

$\int_{S} p_{\theta} d s+\int_{S_{\sigma}}\left(\bar{p}_{\theta}-p_{\theta}\right) d s+\iint_{\Omega} f_{\theta} d \Omega=0$

By means of green-formulation too, one has

$\iint_{\Omega}\left(\frac{\partial \sigma_{r \theta}}{\partial r}+\frac{1}{r} \frac{\partial \sigma_{\theta}}{\partial \theta}+\frac{2 \sigma_{r \theta}}{r}+f_{\theta}\right) d \Omega+\int_{S_{\sigma}}\left(\bar{p}_{\theta}-p_{\theta}\right) d s=0$

In light of definition of in the radial direction strain on $\delta \Omega$, has

$\iint_{\delta \Omega} e_{r} d \Omega=\int_{\delta S} u \cdot n_{r} d s$

when $u=\bar{u}$ on $S_{u}$, then for all area

$\iint_{\Omega} e_{r} d \Omega=\int_{S-S_{u}} u \cdot n_{r} d s+\int_{S_{u}} \bar{u} \cdot n_{r} d s$

yields

$\iint_{\Omega} e_{r} d \Omega=\int_{S} u \cdot n_{r} d s+\int_{S_{u}}(\bar{u}-u) \cdot n_{r} d s$

By means of green-formulation, the radial direction generalized conforming equation as follows

$\iint_{\Omega}\left(\frac{\partial u}{\partial r}-e_{r}\right) d \Omega+\int_{S_{u}}(\bar{u}-u) n_{r} d s=0$

similar to equation (2a), we have below equations

$$
\begin{aligned}
& \iint_{\Omega}\left(\frac{u}{r}+\frac{1}{r} \frac{\partial w}{\partial \theta}-e_{\theta}\right) d \Omega+\int_{S_{u}}(\bar{w}-w) n_{\theta} d s=0 \\
& \iint_{\Omega}\left(\frac{1}{r} \frac{\partial u}{\partial \theta}+\frac{\partial w}{\partial r}-\frac{w}{r}-e_{r \theta}\right) d \Omega+\int_{S_{u}}\left((\bar{u}-u) n_{\theta}+(\bar{w}-w) n_{r}\right) d s=0
\end{aligned}
$$


in which the usual index notation is used. $S_{\sigma}$ and $S_{u}$ denote respectively the portion of the edge boundary where tractions $\bar{p}_{i}$ are prescribed and where displacements $\bar{u}_{i}$ are prescribed. The stress-strain relations of isotropy is (for plane stress problem)

$$
\left\{\begin{array}{c}
\sigma_{r} \\
\sigma_{\theta} \\
\sigma_{r \theta}
\end{array}\right\}=\frac{E}{\left(1-v^{2}\right)}\left[\begin{array}{ccc}
1 & v & 0 \\
v & 1 & 0 \\
0 & 0 & \frac{1-v}{2}
\end{array}\right]\left\{\begin{array}{c}
e_{r} \\
e_{\theta} \\
e_{r \theta}
\end{array}\right\}, \quad\left\{\begin{array}{c}
e_{r} \\
e_{\theta} \\
e_{r \theta}
\end{array}\right\}=\frac{1}{E}\left[\begin{array}{ccc}
1 & -v & 0 \\
-v & 1 & 0 \\
0 & 0 & 2(1+v)
\end{array}\right]\left\{\begin{array}{c}
\sigma_{r} \\
\sigma_{\theta} \\
\sigma_{r \theta}
\end{array}\right\}
$$

For plane strain problem, one has

$$
\left\{\begin{array}{c}
\sigma_{r} \\
\sigma_{\theta} \\
\sigma_{r \theta}
\end{array}\right\}=\frac{E}{(1+v)(1-2 v)}\left[\begin{array}{ccc}
1-v & v & 0 \\
v & 1-v & 0 \\
0 & 0 & \frac{1-2 v}{2}
\end{array}\right]\left\{\begin{array}{c}
e_{r} \\
e_{\theta} \\
e_{r \theta}
\end{array}\right\}
$$

Substituting equation (3) into equations (2a), (2b) and (2c), then integrating by the weight function, i.e. multiply (1a) and (1b) by $\delta u, \delta w$ and multiply (2a)-(2c) by $\delta \sigma_{r}, \delta \sigma_{\theta}, \delta \sigma_{r \theta}$, respectively. One denotes $\boldsymbol{q}=(u, w)^{T}, \boldsymbol{p}=$ $\left(\sigma_{r}, \sigma_{r \theta}\right)^{T}, p_{1}=\sigma_{\theta}, \boldsymbol{F}=\left(\sigma_{r}, \sigma_{r \theta}, u, w\right)^{T}$, weak formulation of mixed state equation including boundary conditions of sectorial domain can be obtained

$$
\begin{aligned}
& \iint_{\Omega} \delta \boldsymbol{F}_{u} \cdot \frac{\partial}{\partial r} \boldsymbol{F} d \Omega=\iint_{\Omega} \delta \boldsymbol{F}_{u} \cdot\left(\boldsymbol{H} \boldsymbol{F}+\boldsymbol{T}+\boldsymbol{D}_{\mathbf{1}} p_{\mathbf{1}}\right) d \Omega+\int_{S} \delta \boldsymbol{F}_{u} \cdot \boldsymbol{S}_{1} d s \\
& \iint_{\Omega} \delta p_{\mathbf{1}} \cdot\left(\boldsymbol{D}_{\mathbf{2}} \boldsymbol{F}+\frac{1}{E} p_{\mathbf{1}}\right) d \Omega+\int_{S} \delta p_{\mathbf{1}} \cdot(w-\bar{w}) n_{\theta} d s=0
\end{aligned}
$$

where

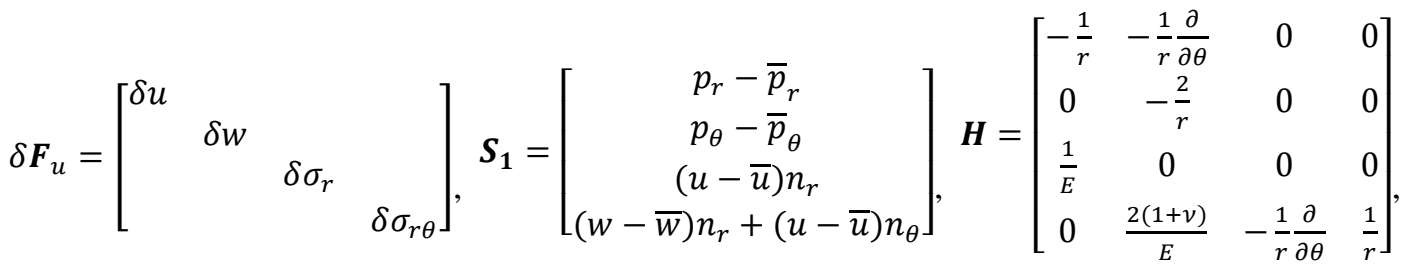

$$
\begin{aligned}
& \boldsymbol{D}_{1}=\left[\begin{array}{c}
\frac{1}{r} \\
-\frac{1}{r} \frac{\partial}{\partial \theta} \\
-\frac{v}{E} \\
0
\end{array}\right], \quad \boldsymbol{D}_{2}=\left[\begin{array}{c}
-\frac{v}{E} \\
0 \\
-\frac{1}{r} \\
-\frac{1}{r} \frac{\partial}{\partial \theta}
\end{array}\right]^{T}, \boldsymbol{T}=\left[\begin{array}{c}
-f_{r} \\
-f_{\theta} \\
0 \\
0
\end{array}\right]
\end{aligned}
$$

\section{Hamilton Equation}

Consider a sectorial domain with two clamped edges $(\theta=0$ and $\theta=\Theta)$. We introduce

$$
\sigma_{\theta}=\sigma_{\bar{\theta}}+U(\theta) a^{(0)}+U(\theta-\Theta) a^{(\Theta)}
$$

$U(\theta), U(\theta-\Theta)$ are unit pulse function, $a^{(0)}, a^{(\Theta)}$ is unknown coefficient at $\theta=0, \Theta$, respectively. (note, we can introduce three different hypothesis for this problem )

Expand the quantities in equations (4) and (5) into the following series system $(\zeta=n \pi / \Theta)$

$$
u=\sum_{n} u_{n}(r) \sin \zeta \theta, \quad w=\sum_{n} w_{n}(r) \cos \zeta \theta, \quad \sigma_{r}=\sum_{n} \sigma_{n}(r) \sin \zeta \theta, \quad \sigma_{r \theta}=\sum_{n} \sigma_{r \theta, n}(r) \cos \zeta \theta
$$

Introducing equations (6) and (7) into equations (4) and (5), after simplifying for each $n$ we obtained (when the body force is zero) 


$$
\begin{aligned}
& \frac{d}{d r}\left[\begin{array}{c}
u_{n}(r) \\
w_{n}(r) \\
\sigma_{n}(r) \\
\sigma_{r \theta, n}(r)
\end{array}\right]=\left[\begin{array}{cccc}
-\frac{v}{r} & \frac{\zeta v}{r} & \frac{1-v^{2}}{E} & 0 \\
-\frac{\zeta}{r} & \frac{1}{r} & 0 & \frac{2(1+v)}{E} \\
\frac{E}{r^{2}} & -\frac{E \zeta}{r^{2}} & \frac{v-1}{r} & \frac{\zeta}{r} \\
-\frac{E \zeta}{r^{2}} & \frac{E \zeta^{2}}{r^{2}} & -\frac{\zeta v}{r} & -\frac{2}{r}
\end{array}\right]\left[\begin{array}{c}
u_{n}(r) \\
w_{n}(r) \\
\sigma_{n}(r) \\
\sigma_{r \theta, n}(r)
\end{array}\right]+\left[\begin{array}{c}
0 \\
0 \\
0 \\
\frac{2}{r \theta}\left(a^{(0)}-(-1)^{n} a^{(\theta)}\right)
\end{array}\right](n \neq 0) \\
& \frac{d}{d r}\left[\begin{array}{c}
u_{n}(r) \\
w_{n}(r) \\
\sigma_{n}(r) \\
\sigma_{r \theta, n}(r)
\end{array}\right]=\left[\begin{array}{cccc}
-\frac{v}{r} & 0 & \frac{1-v^{2}}{E} & 0 \\
0 & \frac{1}{r} & 0 & \frac{2(1+v)}{E} \\
\frac{E}{r^{2}} & 0 & \frac{v-1}{r} & 0 \\
0 & 0 & 0 & -\frac{2}{r}
\end{array}\right]\left[\begin{array}{c}
u_{n}(r) \\
w_{n}(r) \\
\sigma_{n}(r) \\
\sigma_{r \theta, n}(r)
\end{array}\right]+\left[\begin{array}{c}
0 \\
0 \\
0 \\
\frac{1}{r \theta}\left(a^{(0)}-a^{(\theta)}\right)
\end{array}\right](n=0)
\end{aligned}
$$

Transforming equation (8) into Hamilton equation. We can take advantage of Hamilton structures to solve it once entering Hamilton system. In fact, Hamilton canonical equations have been existed in equation (4) impliedly. (see reference, Acta Mechanica Sinica,1998,30(5),580-586,in Chinese)

$$
\begin{aligned}
& \frac{d}{d r}\left[\begin{array}{l}
u_{n}(r) \\
w_{n}(r) \\
\alpha_{n}(r) \\
\beta_{n}(r)
\end{array}\right]=\frac{d}{d r}\left[\begin{array}{c}
u_{n}(r) \\
w_{n}(r) \\
r \sigma_{n}(r) \\
r \sigma_{r \theta, n}(r)
\end{array}\right] \\
& =\left[\begin{array}{cccc}
-\frac{v}{r} & \frac{\zeta v}{r} & \frac{1-v^{2}}{E \cdot r} & 0 \\
-\frac{\zeta}{r} & \frac{1}{r} & 0 & \frac{2(1+v)}{E \cdot r} \\
\frac{E}{r} & -\frac{E \zeta}{r} & \frac{v}{r} & \frac{\zeta}{r} \\
-\frac{E \zeta}{r} & \frac{E \zeta^{2}}{r} & -\frac{\zeta v}{r} & -\frac{1}{r}
\end{array}\right]\left[\begin{array}{l}
u_{n}(r) \\
w_{n}(r) \\
\alpha_{n}(r) \\
\beta_{n}(r)
\end{array}\right]+\left[\begin{array}{c}
0 \\
0 \\
0 \\
\frac{2}{\theta}\left(a^{(0)}-(-1)^{n} a^{(\theta)}\right)
\end{array}\right](n \neq 0) \\
& \frac{d}{d r}\left[\begin{array}{l}
u_{n}(r) \\
w_{n}(r) \\
\alpha_{n}(r) \\
\beta_{n}(r)
\end{array}\right]=\frac{d}{d r}\left[\begin{array}{c}
u_{n}(r) \\
w_{n}(r) \\
r \sigma_{n}(r) \\
r \sigma_{r \theta, n}(r)
\end{array}\right] \\
& =\left[\begin{array}{cccc}
-\frac{v}{r} & 0 & \frac{1-v^{2}}{E \cdot r} & 0 \\
0 & \frac{1}{r} & 0 & \frac{2(1+v)}{E \cdot r} \\
\frac{E}{r} & 0 & \frac{v}{r} & 0 \\
0 & 0 & 0 & -\frac{1}{r}
\end{array}\right]\left[\begin{array}{c}
u_{n}(r) \\
w_{n}(r) \\
\alpha_{n}(r) \\
\beta_{n}(r)
\end{array}\right]+\left[\begin{array}{c}
0 \\
0 \\
0 \\
\frac{1}{\theta}\left(a^{(0)}-a^{(\theta)}\right)
\end{array}\right](n=0)
\end{aligned}
$$

Let us simply equation (9),

$$
\frac{d}{d r} \boldsymbol{\Psi}_{1}=\boldsymbol{M}_{1} \cdot \boldsymbol{\Psi}_{1}+\boldsymbol{\Lambda}_{1}
$$

in which

$$
\begin{aligned}
& \boldsymbol{\Psi}_{\mathbf{1}}=\left[\begin{array}{llll}
u_{n}(r) & w_{n}(r) & \alpha_{n}(r) & \beta_{n}(r)
\end{array}\right]^{T}, \\
& \boldsymbol{M}_{1}=\left[\begin{array}{cc}
\boldsymbol{A}_{1} & \boldsymbol{G}_{1} \\
\boldsymbol{B}_{1} & -\boldsymbol{A}_{1}{ }^{T}
\end{array}\right], \boldsymbol{A}_{1}=\left[\begin{array}{cc}
-\frac{v}{r} & \frac{\zeta v}{r} \\
-\frac{\zeta}{r} & \frac{1}{r}
\end{array}\right], \boldsymbol{G}_{1}=\left[\begin{array}{cc}
\frac{1-v^{2}}{E \cdot r} & 0 \\
0 & \frac{2(1+v)}{E \cdot r}
\end{array}\right], \boldsymbol{B}_{1}=\left[\begin{array}{cc}
\frac{E}{r} & -\frac{E \zeta}{r} \\
-\frac{E \zeta}{r} & \frac{E \zeta^{2}}{r}
\end{array}\right], \quad-\boldsymbol{A}_{1}{ }^{T}=\left[\begin{array}{cc}
\frac{v}{r} & \frac{\zeta}{r} \\
-\frac{\zeta v}{r} & -\frac{1}{r}
\end{array}\right] \\
& \Lambda_{1}=\left[\begin{array}{llll}
0 & 0 & 0 & \frac{2}{\Theta}\left(a^{(0)}-(-1)^{n} a^{(\Theta)}\right)
\end{array}\right]^{T}(n \neq 0), \quad \Lambda_{1}=\left[\begin{array}{llll}
0 & 0 & 0 & \frac{1}{\Theta}\left(a^{(0)}-a^{(\Theta)}\right)
\end{array}\right]^{T} \quad(n=0)
\end{aligned}
$$


$\boldsymbol{M}_{1}$ in equation (10) is Hamilton matrix. Due to equation (10) is variable-coefficient differential equation, it is inconvenient to solve it. We can change it into ordinary differential equation by variable substitution, letting $\eta=$ $\ln r, r=e^{\eta}$, one has

$$
\frac{d}{d \eta} \boldsymbol{\Psi}=\boldsymbol{M} \cdot \boldsymbol{\Psi}+\boldsymbol{\Lambda}
$$

in which

$$
\begin{aligned}
& \boldsymbol{\Psi}=\left[\begin{array}{llll}
u_{n}(\eta) & w_{n}(\eta) & \alpha_{n}(\eta) & \beta_{n}(\eta)
\end{array}\right]^{T}, \\
& \boldsymbol{M}=\left[\begin{array}{cc}
\boldsymbol{A} & \boldsymbol{G} \\
\boldsymbol{B} & -\boldsymbol{A}^{\boldsymbol{T}}
\end{array}\right], \boldsymbol{A}=\left[\begin{array}{cc}
-v & \zeta v \\
-\zeta & 1
\end{array}\right], \boldsymbol{G}=\left[\begin{array}{cc}
\frac{1-v^{2}}{E} & 0 \\
0 & \frac{2(1+v)}{E}
\end{array}\right], \boldsymbol{B}=\left[\begin{array}{cc}
E & -E \zeta \\
-E \zeta & E \zeta^{2}
\end{array}\right],-\boldsymbol{A}^{T}=\left[\begin{array}{cc}
v & \zeta \\
-\zeta v & -1
\end{array}\right] \\
& \boldsymbol{\Lambda}=\left[\begin{array}{llll}
0 & 0 & 0 & \frac{2 e^{\eta}}{\Theta}\left(a^{(0)}-(-1)^{n} a^{(\Theta)}\right)
\end{array}\right]^{T}(n \neq 0), \quad \boldsymbol{\Lambda}=\left[\begin{array}{llll}
0 & 0 & 0 & \frac{e^{\eta}}{\Theta}\left(a^{(0)}-a^{(\Theta)}\right)
\end{array}\right]^{T} \quad(n=0)
\end{aligned}
$$

Thus all terms of Hamilton matrix are constants.

\section{Solution}

The solution of equation (11) is

$$
\boldsymbol{\Psi}\left(\eta_{a}\right)=\exp \left[\boldsymbol{M} \cdot\left(\eta_{b}-\eta_{a}\right)\right] \boldsymbol{\Psi}\left(\eta_{b}\right)+\int_{\eta_{b}}^{\eta_{a}} \exp [\boldsymbol{M} \cdot(\eta-\tau)] \cdot \boldsymbol{\Lambda}(\tau) d \tau
$$

In order to calculate the above matrix function, the eigenvalues of the matrix $\boldsymbol{M}$ must be considered. Because $\boldsymbol{M}$ is Hamilton matrix, its eigenvalues must appear in pairs.

So far, we still haven't known that distribution of unknown coefficients along $r$ direction, in order to solve it, we may divide longitudinal thick into some minor thin plies, for example $m$ thin plies. Of course, we have reason to think unknown coefficient within each minor thin ply is constant. If we find, from calculation, that the needful effective digits hardly change, it can be said that the results obtained with some minor thin plies are exact within the prescribed accuracy limits. After programming, one has

$$
\boldsymbol{\Psi}\left(\eta_{a}\right)=\boldsymbol{C} \cdot \boldsymbol{\Psi}\left(\eta_{b}\right)+\boldsymbol{D} \cdot \boldsymbol{\Lambda}
$$

where $\boldsymbol{D}$ is a matrix of $4 \times m$, and $\boldsymbol{\Lambda}$ is a contains unknown coefficients matrix of $m \times 1$. Usually, the loads on the inner and outer surface of sector domain are given a priori. We can obtain below equation from equation (13)

$$
\begin{aligned}
& {\left[\begin{array}{l}
\alpha\left(\eta_{a}\right) \\
\beta\left(\eta_{a}\right)
\end{array}\right]=\left[\begin{array}{ll}
C^{31} & C^{32} \\
C^{41} & C^{42}
\end{array}\right] \cdot\left[\begin{array}{l}
u\left(\eta_{b}\right) \\
w\left(\eta_{b}\right)
\end{array}\right]+\left[\begin{array}{ll}
C^{33} & C^{34} \\
C^{43} & C^{44}
\end{array}\right] \cdot\left[\begin{array}{l}
\alpha\left(\eta_{b}\right) \\
\beta\left(\eta_{b}\right)
\end{array}\right]+\left[\begin{array}{l}
D^{(3)} \\
D^{(4)}
\end{array}\right] \cdot \boldsymbol{\Lambda}} \\
& {\left[\begin{array}{l}
u\left(\eta_{b}\right) \\
w\left(\eta_{b}\right)
\end{array}\right]=\left[\begin{array}{ll}
C^{31} & C^{32} \\
C^{41} & C^{42}
\end{array}\right] \cdot\left(\left[\begin{array}{l}
\alpha\left(\eta_{a}\right) \\
\beta\left(\eta_{a}\right)
\end{array}\right]-\left[\begin{array}{ll}
C^{33} & C^{34} \\
C^{43} & C^{44}
\end{array}\right] \cdot\left[\begin{array}{l}
\alpha\left(\eta_{b}\right) \\
\beta\left(\eta_{b}\right)
\end{array}\right]-\left[\begin{array}{l}
D^{(3)} \\
D^{(4)}
\end{array}\right] \cdot \boldsymbol{\Lambda}\right)}
\end{aligned}
$$

Extending above the second equation, therefore one has

$$
\boldsymbol{\Psi}\left(\eta_{b}\right)=\boldsymbol{C}_{\mathbf{1}}+\boldsymbol{D}_{\mathbf{1}} \cdot \boldsymbol{\Lambda}
$$

in which $\boldsymbol{C}_{\mathbf{1}}, \boldsymbol{D}_{\mathbf{1}}$ are given by $\boldsymbol{C}$ and $\boldsymbol{D}$.

Substituting equation (14) into equation (13),

$$
\boldsymbol{\Psi}\left(\eta_{a}\right)=\boldsymbol{C} \cdot \boldsymbol{C}_{\mathbf{1}}+\left(\boldsymbol{C} \cdot \boldsymbol{D}_{1}+\boldsymbol{D}\right) \cdot \boldsymbol{\Lambda}
$$

for any $\eta$ one has

$$
\boldsymbol{\Psi}(\eta)=\boldsymbol{C}_{\eta} \cdot \boldsymbol{C}_{\mathbf{1}}+\left(\boldsymbol{C}_{\eta} \cdot \boldsymbol{D}_{1}+\boldsymbol{D}_{\eta}\right) \cdot \boldsymbol{\Lambda}
$$


where $\boldsymbol{C}_{\eta}$ and $\boldsymbol{D}_{\eta}$ can be calculated through matrix function of equation (12). From equation (16), there are only unknown quantities left here, they can be got by means of satisfying boundary condition (the present approach). In addition, owing to each mechanics quantity is expressed by $\boldsymbol{\Lambda}$, they can also be calculated by fractal finite element method. By restoring variable substitution, from equation (16), and has

$$
\left\{\begin{array}{c}
\sigma_{r} \\
\sigma_{r \theta}
\end{array}\right\}=\frac{1}{r}\left\{\begin{array}{l}
\Psi^{(3)}(\eta) \\
\Psi^{(4)}(\eta)
\end{array}\right\}
$$

where $\Psi^{(3)}(\eta)$ and $\Psi^{(4)}(\eta)$ come from the third and fourth row of equation (16). We know stresses have singularity when $r \rightarrow 0$ from equations (17).

According to knowledge of fractal finite element method, within the singular region, one can use fractal twolevel finite element method to solve unknown coefficient. The second level interpolation of displacements for the first layer of fractal mesh can be written as follows

$$
\left\{\begin{array}{l}
\boldsymbol{v}_{\boldsymbol{m}} \\
\boldsymbol{v}_{\boldsymbol{s}}
\end{array}\right\}=\left[\begin{array}{cc}
\boldsymbol{I} & \mathbf{0} \\
\mathbf{0} & \boldsymbol{T}_{s}^{f}
\end{array}\right]\left\{\begin{array}{c}
\boldsymbol{v}_{m} \\
\boldsymbol{\Lambda}
\end{array}\right\}
$$

where $\boldsymbol{v}_{\boldsymbol{r}}, \boldsymbol{v}_{m}$ are the displacements in the regular region and on the boundary respectively. $\boldsymbol{I}$ is the identity matrix, $\boldsymbol{T}_{s}^{f}$ is the transformation matrix that can be evaluated by using equations (7) and (15). Applying the fractal transformation technique (18), the global stiffness matrix becomes

$$
\left[\begin{array}{ccc}
K_{r r} & K_{r m} & \\
K_{m r} & K_{m m}+K_{11}^{1} & K_{12}^{1} T_{1} \\
& K_{21}^{1} T_{1} & K_{s s}
\end{array}\right]\left[\begin{array}{c}
v_{r} \\
v_{m} \\
\Lambda
\end{array}\right]
$$

From equation (19), after the transformation, the order of equation (19) becomes much smaller than that of equation (18). When unknown coefficient is obtained, this problem is achieved.

\section{Conclusion}

Weak formulations of mixed state equations are presented in Hamilton System. This work is concerned with the weak formulation of mixed equation of elasticity and the fractal finite element methods. By means of the weak formulation, some analytical solutions expression can be obtained. The fractal finite element method is used to analyze the sectorial domain problem. The present study satisfies the continuity conditions of stresses and displacements at the interfaces. Solutions and method such as this have value for designing laminated composite structures in naval, aerospace and other engineering applications.

\section{Acknowledgments}

This project 11472005 supported by National Natural Science Foundation of China.

\section{References}

[1] Zienkiewicz R L, Taylor. The finite element method. NewYork, Mcgraw-Hill Book Company, 1988.

[2] Pian T H H, Chen D P. On the suppression of zero energy deformation modes. International Journal for Numerical Methods in Engineering, 1983, 19(12): 1741-1752.

[3] Tang L, Chen W, Liu Y. String net function approximation and quasi-conforming technique, hybid and mixed finite element methods. Atluri S N, Gallagher R H, Zienkiewicz O C, translated. New York: John Wiley \& Sons, 1983.

[4] Tang L, Chen W, Liu Y. Formulation of quasi-conforming element and Hu-Washizu principle. Computers \& Structures, 1984, 19(1): 247-250.

[5] Zhang Hongqing, Wang Ming, FiniChen Wanji. More on the quasi-conforming element methods and the generalized variational principles. Acta MechanicaSolidaSinica, 1983(1): 129-135.(in Chinese)

[6] Zhang Hongqing, Wang Ming. Finite element approximations with multiple sets of functions and quasiconforming elements for plate bending problems. Applied Mathematics and Mechanics, 1985(1): 41-52.(in Chinese) 
[7] Long Yuqiu, Xin Kegui. Generalized conforming element. China Civil Engineering Journal, 1987(1): 1-14.(in Chinese)

[8] Leung AYT, Su RKL. Fractal two-level finite element method for free vibration of cracked beams. Shock \& Vibration, 2013, 5(1): 61-68.

[9] Su RKL, Leung AYT. Three-dimensional mixed mode analysis of a cracked body by fractal finite element method. International Journal of Fracture, 2001, 110(1): 1-20.

[10] Ding Kewei, Tang Limin. Weak formulation of mixed state equation and boundary value problem of theory of elasticity. Acta Mechanica Sinica, 1998, 30(5): 580-586. (in Chinese)

[11] Ding Kewei, Tang Limin. Exact solution for axisymmetric thick laminated shells. Composite Structures, 1999, 46(2): 125-129.

[12] Ding Kewei. Weak formulation study for thermoelastic analysis of thick open laminated shell. Mechanics of Advanced Materials and Structures, 2008, 15(15): 33-39.

[13] Ding Kewei. Weak Formulation Study For Thermoelastic Buckling Analysis Of Thick Laminated Cylindrical Shells. International Journal of Applied Mechanics \& Engineering, 2015, 20(3): 461-470.

[14] Ding Kewei. The Symplectic Algorithm Method for The Weak Formulation Equations of quasi-conforming element. Chinese Journal of Applied Mechanics, 2017, 34(1): 174-179. (in Chinese)

[15] Ding Kewei. Symplectic algorithm for weak formulation of Hamiltonian. Journal of Hefei University of Technology, 2018, 41(1): 71-75. (in Chinese) 